\title{
ASPECTOS REPRODUTIVOS DO PEIXE Lutjanus synagris (PERCIFORMES, LUTJANIDAE) CAPTURADO NA COSTA NORDESTE DO BRASIL
}

\author{
Ana Flavia Ribeiro SOUSA ${ }^{1 *}$; Nayara Barbosa SANTOS ${ }^{2}$; Raimunda Nonata Fortes CARVALHO \\ NETA $^{2}$ \& Zafira da Silva de ALMEIDA ${ }^{2}$ \\ ${ }^{1}$ Curso de Ciências Biológicas, Universidade Estadual do Maranhão-UEMA \\ ${ }^{2}$ Departamento de Química e Biologia, Universidade Estadual do Maranhão-UEMA \\ *email: fa-fa_sousa@hotmail.com \\ Recebido em (30/01/2017)
}

RESUMO - Neste trabalho objetivou-se descrever aspectos da biologia reprodutiva do Lutjanus synagris oriundos da pesca artesanal realizada pela maior comunidade pesqueira do Maranhão, na costa nordeste do Brasil. Os dados foram coletados através de amostragens bimestrais durante os períodos de março de 2013 a fevereiro de 2014. Foram obtidos dados biométricos dos peixes (comprimento e peso), sendo identificado o sexo e o estádio de maturação gonadal. Analisou-se a relação peso-comprimento, a proporção sexual, a época de desova, o comprimento de primeira maturação e a fecundidade. Os peixes analisados variaram de 20 a $48 \mathrm{~cm}$ de comprimento, apresentando alometria negativa para sexos agrupados e proporção sexual de 4,76F: 1M. A primeira maturação ocorreu em fêmeas com 24,02 cm e em machos com 23,59 cm. Os exemplares apresentaram todos os estádios maturacionais, os quais foram confirmados pelas análises macroscópica e microscópica das gônadas. A espécie apresenta desova parcelada com dois picos anuais. A média da fecundidade absoluta foi 709.271 ovócitos por desova. A análise da relação gonadossomática, fator de condição e frequência dos estádios maturacionais apontaram que a espécie está apta a se reproduzir o ano todo, pois foram encontradas fêmeas maturas e desovadas, durante todo o período analisado.

Palavras-Chave: Relação Gonadossomática, Desova, Ariacó

\section{REPRODUCTIVE ASPECTS OF THE FISH LUTJANUS SYNAGRIS (PERCIFORMES, LUTJANIDAE) CAUGHT ON THE COAST OF NORTHEAST BRAZIL}

ABSTRACT - In this work we aimed to describe aspects of reproductive biology of Lutjanus synagris originating from the artisanal fisheries conducted by larger fishing community of Maranhão, in the northeast coast of Brazil. Data were collected through bimonthly sampling during periods of March 2013 to February 2014. Biometric data of fish were obtained (length and weight) with identification of sex and gonadal maturation stage. We analyzed the length-weight relationship, sex ratio, the spawning season, the length of first maturity and fertility. The analyzed fish range from 20 to $48 \mathrm{~cm}$ in length, with negatively allometric for grouped sexes and sexual ratio 4,76F: 1M. The first maturity occurred in females with $24.02 \mathrm{~cm}$ and $23.59 \mathrm{~cm}$ in males. Specimens showed all maturational stages, which were confirmed by macroscopic and microscopic analysis of gonads. The species has multiple spawner with two annual peaks. The mean absolute fecundity was 709271 oocytes per spawning. The analysis of gonadosomatic index, condition factor and frequency of maturation stages showed that the species is able to reproduce all year, as mature and spawned females were found throughout the study period.

Keywords: Gonadosomatic index, Spawning, Lane snapper 
Trabalho financiado por Fundação de Amparo à Pesquisa e Desenvolvimento Científico do Maranhão - FAPEMA. 


\section{INTRODUÇÃO}

Lutjanus synagris (Linnaeus, 1758), popularmente conhecido por ariacó, é um peixe da família Lutjanidae. Geograficamente, tem uma distribuição que ocorre desde a Carolina do Norte, EUA até a região Sudeste do Brasil (Figueiredo \& Menezes, 2000), sendo comumente encontrado em ambientes recifais e bancos de algas até 400 metros de profundidade (Mceachran \& Fechhelm, 2005). No Brasil, a espécie é relativamente abundante na costa Norte e Nordeste, onde, apresenta grande importância comercial (CAVALCANTE, OLIVEIRA \& CHELLAPPA, 2012).

Ao longo da costa tropical brasileira ocorrem 15 espécies de lutjanídeos (MENEZES, BUCKUP, FIGUEIREDO \& MOURA, 2003), chamados de "vermelhos", "pargos" ou “ariacó" e são comumente explorados pelos pescadores artesanais (FRÉDOU, FERREIRA \& LETOURNNEUR, 2006), por possuírem uma carne de qualidade, o que confere às espécies um alto valor econômico (Nelson, 1994; Begossi et al., 2011). E apesar dessa enorme diversidade e importância econômica, dados sobre a dinâmica populacional de várias espécies de Lutjanidae são escassos. No Maranhão a situação se agrava, já que não existem estudos aprofundados sobre ecologia e pesca do grupo em questão, carecendo-se de estudos sobre potencial pesqueiro e aspectos biológicos desses táxons, em especial os relacionados à biologia reprodutiva. A produção pesqueira do ariacó no Brasil decresceu no período de 2007-2011 (BRASIL, 2010, 2011; IBAMA, 2008), indicando a necessidade de políticas de conservação da espécie baseadas em dados científicos.

O estudo da biologia reprodutiva proporciona uma análise mais segura sobre o potencial pesqueiro do ambiente, embora seja necessário o conhecimento de vários outros fatores que influenciam no comportamento das espécies. Informações sobre o desenvolvimento cíclico das gônadas, época e local de desova e comprimento em que os indivíduos entram no processo reprodutivo fazem parte do conhecimento acerca da biologia reprodutiva de uma espécie (SOUZA, CHELLAPPA \& GURGEL, 2007). Estas informações são subsídios necessários e importantes para procedimentos de manejo e conservação de populações nativas e para a regulamentação da pesca (KING, 1995). Assim, é importante acompanhar o ciclo reprodutivo, conhecer e avaliar o início e continuidade do crescimento gonadal assim como o desenvolvimento pleno das gônadas até a maturação final (desova e fertilização) e regressão gonadal (VAZZOLER, 1996).

O ciclo reprodutivo dos lutjanídeos da Costa Maranhense não tem sido estudado adequadamente, embora espécies como Lutjanus synagris seja frequentemente capturada ao longo de 
toda região costeira do Maranhão, especialmente pela comunidade pesqueira da Raposa - MA. Nessa região, a pesca de L. synagris tem grande apelo social, econômico e ecológico e merece especial atenção. Isso porque já existe a percepção ambiental dos pescadores sobre o decréscimo desse pescado e já tem sido estabelecida uma forte demanda da própria comunidade para conhecer os parâmetros reprodutivos essenciais para a determinação de instrumentos de gestão, como o período de defeso da espécie (IBAMA, 2010). Neste contexto, o presente estudo visou descrever aspectos da biologia reprodutiva do Lutjanus synagris oriundos da pesca artesanal realizada pela maior comunidade pesqueira do Maranhão (município de Raposa), na costa nordeste do Brasil.

\section{MAterial e Métodos}

\section{OBTENÇÃO DE DADOS}

O material para a realização desse estudo foi proveniente da pesca comercial na região costeira nas proximidades do município da Raposa ( $02^{\circ} 25^{\prime} 22^{\prime \prime S}$ e $\left.44^{\circ} 05^{\prime} 21^{\prime \prime W}\right)$, envolvendo parte da Baía de São Marcos e parte da Baía de São José (Figura 1). Essa comunidade pertence à Região Metropolitana de São Luís e é banhada pelo Oceano Atlântico. Segundo dados do IBGE (2014), esse município apresenta uma população de 29.167 habitantes dos quais mais de $80 \%$ tem na pesca a sua principal ocupação.

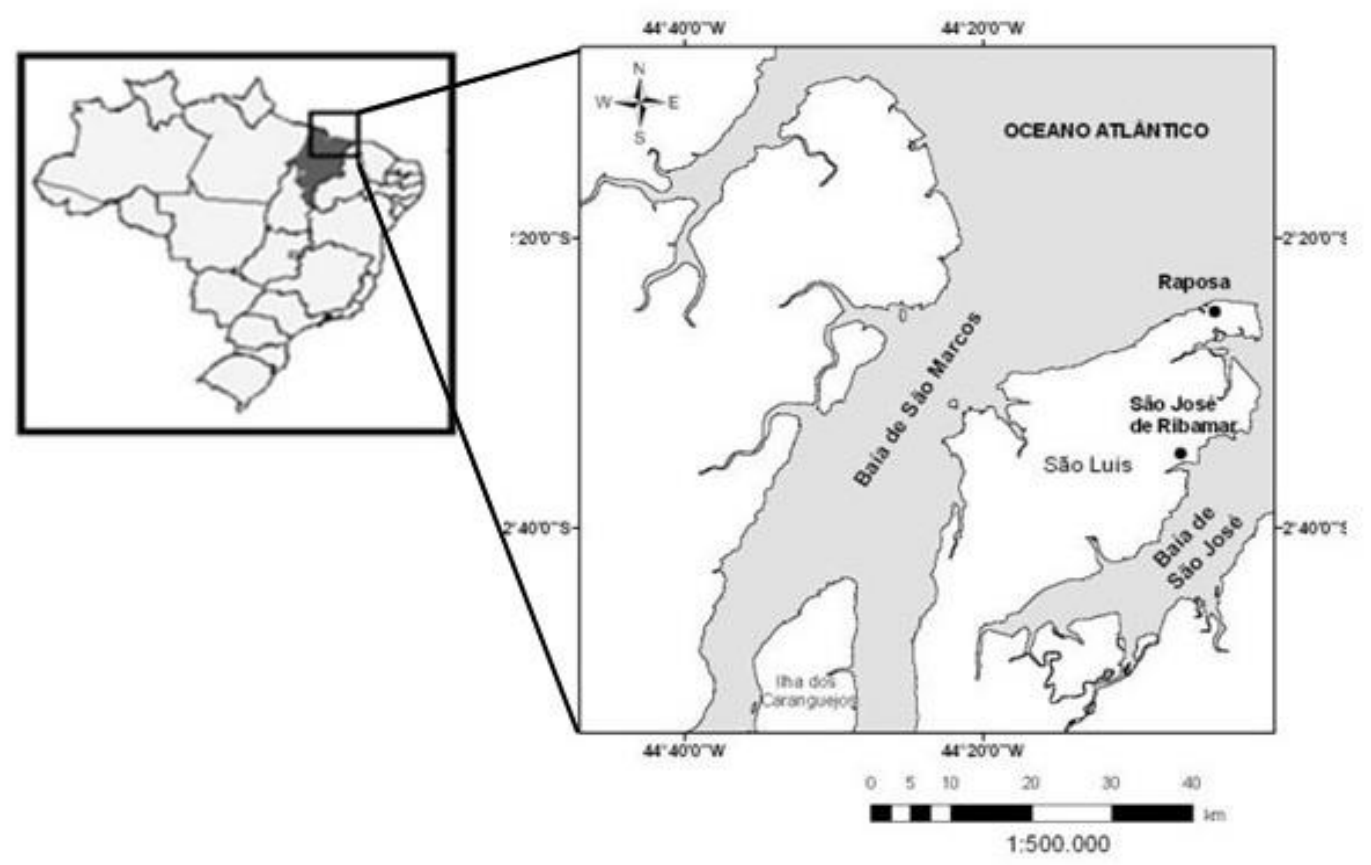

Figura 1. Golfão maranhense com destaque para a comunidade pesqueira da Raposa. 
Os peixes foram capturados com o auxílio dos pescadores que utilizaram rede de emalhar, e pela frota do município da Raposa. A compra dos exemplares ocorreu a cada dois meses, e foi previamente encomendada de forma que os peixes escolhidos atendessem a uma ampla faixa de comprimento, para que essa amostragem tivesse indivíduos, em todas as fases de desenvolvimento reprodutivo. Foram analisados aproximadamente 40 exemplares de L. synagris por bimestre. Esses peixes foram analisados no Laboratório de Pesca e Ecologia Aquática da Universidade Estadual do Maranhão - UEMA, onde foram previamente identificados, contados e pesados. Registrou-se o comprimento total $(\mathrm{cm})$, comprimento padrão $(\mathrm{cm})$ e peso total $(\mathrm{g})$, sexo e estádios maturacionais.

\section{ANALISE DOS DADOS}

Os estádios maturacionais e o sexo de L. synagris foram determinados através de análises macroscópicas e confirmados com análise histológica quando necessário. Em cada exemplar foi realizada uma secção ventro-longitudinal para extração das gônadas e identificação macroscópica, observando-se algumas características como: cor, vascularização, volume em relação à cavidade abdominal, irrigação sanguínea, visibilidade dos ovócitos, presença de esperma e sua consistência. Uma escala de maturação previamente estabelecida foi utilizada para classificação macroscópica das gônadas, conforme as seguintes categorias: $\mathrm{A}=$ imaturo; $\mathrm{B}=$ em maturação; $\mathrm{C}=$ maturo e $\mathrm{D}=$ desovado/esvaziado (VAZZOLER, 1996). Em seguida foram pesadas em balança de precisão de 0,01g.

Para análise microscópica, as gônadas foram seccionadas em três partes (proximal, mediana e distal) e as porções medianas foram fixadas em Bouin, com inclusão em parafina e coloração utilizando método de hematoxilina-eosina (HE).

A relação entre comprimento total e peso total foi estabelecida através da regressão não linear. $\mathrm{O}$ ajuste da curva representada pela expressão matemática, $\mathrm{PT}=\mathrm{a} \times \mathrm{CT}^{b}$, foi obtida pelo método dos mínimos quadrados Zar (1996), onde PT é o peso total do peixe, a é o coeficiente linear de regressão, CT é o comprimento total do peixe e b é o coeficiente angular de regressão.

A proporção sexual dos indivíduos amostrados foi obtida para o período total e por classes de comprimento. Para verificar a existência de diferenças significativas na proporção entre os sexos foi utilizado o teste do $\chi^{2}$ com correção de Yates com nível de significância de $5 \%$ definido pela equação.

Para a análise do tamanho da primeira maturação (L50), os estádios maturacionais foram agrupados em imaturos (estádio A) e em maturos (estádios $\mathrm{B}+\mathrm{C}+\mathrm{D}$ ), seguindo o proposto por 
Vazzoler (1996) e Ortiz-Ordónez et al. (2007). O percentual de maturos por classe de comprimento foi calculado e considerado como variável dependente (Y) e o comprimento total como variável independente $(\mathrm{X})$. Posteriormente, estes valores foram ajustados a uma curva logística utilizando o software Statistica, versão 6.0, segundo a fórmula: $\mathrm{P}=1 /(1+\exp [-\mathrm{r}(\mathrm{CT}-\mathrm{L} 50)])$, onde: $\mathrm{P}=$ proporção de indivíduos maturos; $\mathrm{r}=$ declive da curva; CT = Comprimento total; $\mathrm{L} 50=$ comprimento médio de maturidade sexual.

O período reprodutivo foi definido com base na frequência bimestral dos estádios de maturidade e pela variação dos valores médios da relação gonadossomática ( $\Delta \mathrm{RGS})$ e do fator de condição $(\Delta \mathrm{K})$.

A relação gonadossomática $(\Delta \mathrm{RGS})$ é a diferença entre RGS1 e RGS2 (índice gonadossomático), dado de acordo com as fórmulas:

RGS1 $=(\mathrm{Pg} / \mathrm{PT}) \times 100$

$\mathrm{RGS} 2=(\mathrm{Pg} / \mathrm{Pc}) \mathrm{x} 100$

Onde: $\mathrm{Pg}=$ peso da gônada; $\mathrm{PT}=$ peso total do indivíduo; $\mathrm{Pc}=\mathrm{PT}-\mathrm{pg}$.

O fator de condição $(\Delta K)$ é a diferença entre os dois modelos $K 1$ e K2 (índices do fator de condição alométrico), dado de acordo com as fórmulas:

$\mathrm{K} 1=\mathrm{PT} / \mathrm{CT}^{\mathrm{b}}$

$\mathrm{K} 2=\mathrm{PC} / \mathrm{CT}^{\mathrm{b}}$

Onde: $\mathrm{K} 1$ = fator de condição total; $\mathrm{K} 2$ = fator de condição somático; $\mathrm{PT}=$ peso total do indivíduo; $\mathrm{CT}=$ comprimento total do indivíduo; $\mathrm{b}=$ coeficiente angular da relação peso/ comprimento; $\mathrm{PC}=\mathrm{PT}-\mathrm{Pg}$, em que: $\mathrm{Pg}=$ peso da gônada.

Para a determinação da fecundidade $(\mathrm{F})$ foi utilizado o método volumétrico (Vazzoler, 1981; 1996) para as gônadas de fêmeas em estágio maduro. A massa total dos ovócitos dissociados suspensos em álcool $70 \%$ foi colocada em uma proveta para a determinação do volume total da suspensão (S). Após 24 horas de sedimentação obteve-se o volume total de ovócitos (V) de cada gônada. A esse volume foi acrescentada a quantidade de álcool $70 \%$ necessária para retirar completamente os ovócitos das paredes da proveta durante a transferência do material para um recipiente, corrigindo-se, assim, o valor de $\mathbf{S}$, que foi posteriormente retirada das subamostras.

$\mathrm{O}$ número total de ovócitos contido nos ovários foi estimado pela expressão $\mathrm{N}^{\prime}=(\mathrm{S} \times \mathrm{n}) / \mathrm{s}$, sendo: N'o número total de ovócitos, S o volume da suspensão, n o número de ovócitos na suspensão pipetada e s o volume da suspensão pipetada $(1 \mathrm{~mL})$. A fecundidade total ou absoluta foi definida como sendo o número de ovócitos maduros e capazes de serem eliminados no período reprodutivo, sendo 
estimada por $\mathrm{N}=\left(\mathrm{N}^{\prime} \times \mathrm{P}\right) / 100$, onde: $\mathrm{N}$ a fecundidade absoluta ou individual, $\mathrm{N}^{\prime}$ o número de ovócitos contidos nos ovários e $\mathrm{P}$ a porcentagem de ovócitos considerados maduros.

\section{RESULTADOS}

Foram analisados 234 exemplares de L. synagris com comprimento total entre 20,0 e 48,0 cm. Quanto à análise de distribuição de frequência por sexo, constatou-se maior frequência de machos na classe de comprimento de 34, a 36,0 cm e de fêmeas na classe entre 30,0 a 32,0 cm (Figura 2).

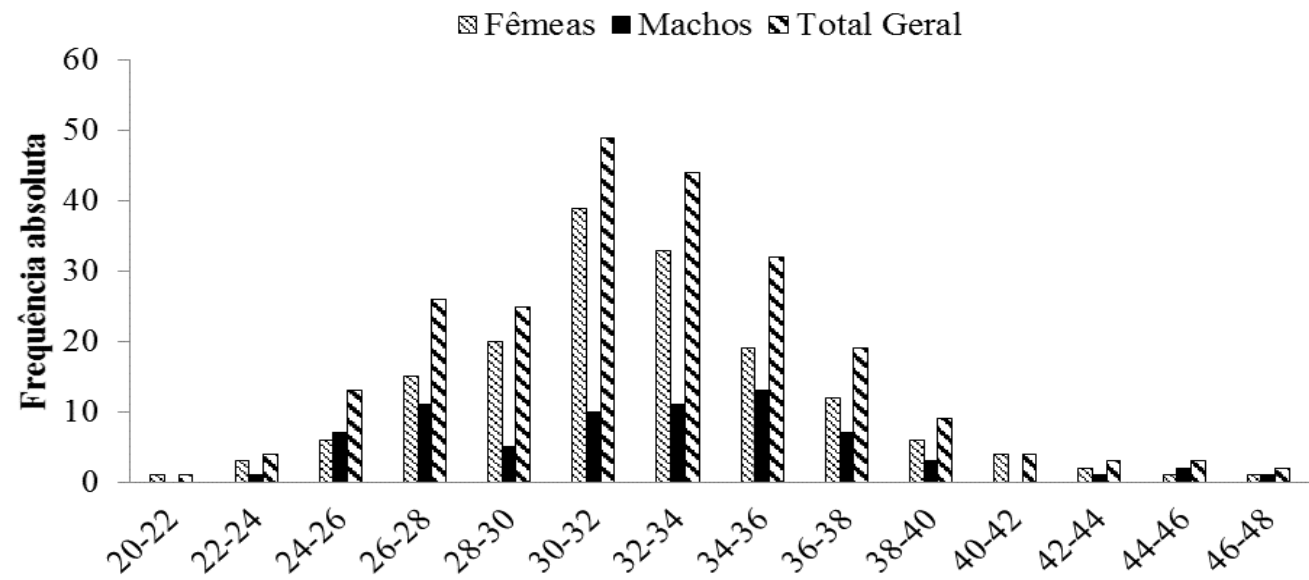

Figura 2. Distribuição da frequência absoluta por classe de comprimento total dos sexos de $L$. synagris.

A alometria foi do tipo negativa para ambos os sexos, indicando que a espécie no local estudado está ganhando mais incremento em comprimento do que em peso. O coeficiente angular de regressão (b) não apresentou diferenças significativas entre machos e fêmeas $(t=0,3858 ; p>0,05)$.

Tabela 1. Relação entre peso total e comprimento total para fêmeas e machos de L. synagris, capturados nas proximidades da Raposa-MA de março de 2013 a fevereiro de 2014. (n= numero de individuos; $\mathrm{CT}=$ comprimento; $\mathrm{A}=$ coeficiente linear; $\mathrm{b}=$ coeficiente de regressão; $r^{2}=$ coeficiente de determinação).

\begin{tabular}{ccccccc}
\hline & $\mathrm{n}$ & $\begin{array}{c}\text { CT (Min-Max) } \\
(\mathrm{cm})\end{array}$ & $\mathrm{A}$ & $\mathrm{b}$ & $\mathrm{r}^{2}$ & Alometria \\
\hline Fêmeas & 162 & $20,0-48,0$ & 0,0396 & 2,709 & 0,86 & Negativa \\
Machos & 72 & $22,0-48,0$ & 0,0774 & 2,5175 & 0,84 & Negativa \\
\hline
\end{tabular}


Quanto à proporção sexual por período total para L. synagris não foi equitativa entre os sexos, 162 fêmeas e 34 machos, sendo favorável para as fêmeas (4,76F: 1M) $\left(\chi^{2}=33,09\right.$; $\left.\mathrm{p}<0,05\right)$. Considerando o período de amostragem, ocorreu dominância das fêmeas tanto na estação chuvosa (Março a Junho/Janeiro e Fevereiro) (1,63F:1M) $\left(\chi^{2}=10,68\right.$; p<0,05), quanto na estação de estiagem (Julho a Dezembro) $(3,21 \mathrm{~F}: 1 \mathrm{M}) \quad\left(\chi^{2}=34,15 ; \mathrm{p}<0,05\right)$. O teste do $\chi^{2}$ apontou existir diferenças significativas entre machos e fêmeas nos três casos.

As fêmeas predominaram em quase todos os bimestres, com exceção do bimestre Mar/Abr em que houve um número maior de exemplares machos. Porém, apesar desta predominância, o teste do $\chi^{2}$ $(0,02 ; \mathrm{p}<0,05)$ indicou que não houve diferenças significativas entre os sexos para este bimestre, diferindo dos outros bimestres onde as fêmeas predominaram.

Considerando a proporção sexual por classe de comprimento, observou-se que a maioria dos indivíduos apresentou comprimentos entre 30 e $32 \mathrm{~cm}$, sendo encontrado maior percentual de fêmeas nas classes de comprimento 30 a $32 \mathrm{~cm}$, com diferença estatística significativa. Os machos tiveram maior frequência proporcional nas classes 34 a 36 não havendo diferença significativa. O teste do $\chi^{2}$ demonstrou que para todas as classes de comprimento, com exceção de $28-30 \mathrm{~cm}$ e $30-32 \mathrm{~cm}$, não houve diferenças significativas entre os sexos.

O tamanho de primeira maturidade sexual de L. synagris para o período estudado foi de 24,02 cm para fêmeas e $23,59 \mathrm{~cm}$ para machos e $23,14 \mathrm{~cm}$ para sexos agrupados, ou seja, o tamanho mínimo de captura, onde $50 \%$ da população estão aptas a se reproduzir.

A partir das observações macroscópicas das gônadas constatou-se indivíduos de L. synagris nos quatro estádios de maturação: Imaturo (A), em maturação (B), maturo (C) e desovado (D) (Figura 3 e Tabela 2), confirmados através de lâminas histológicas (Figura 4).
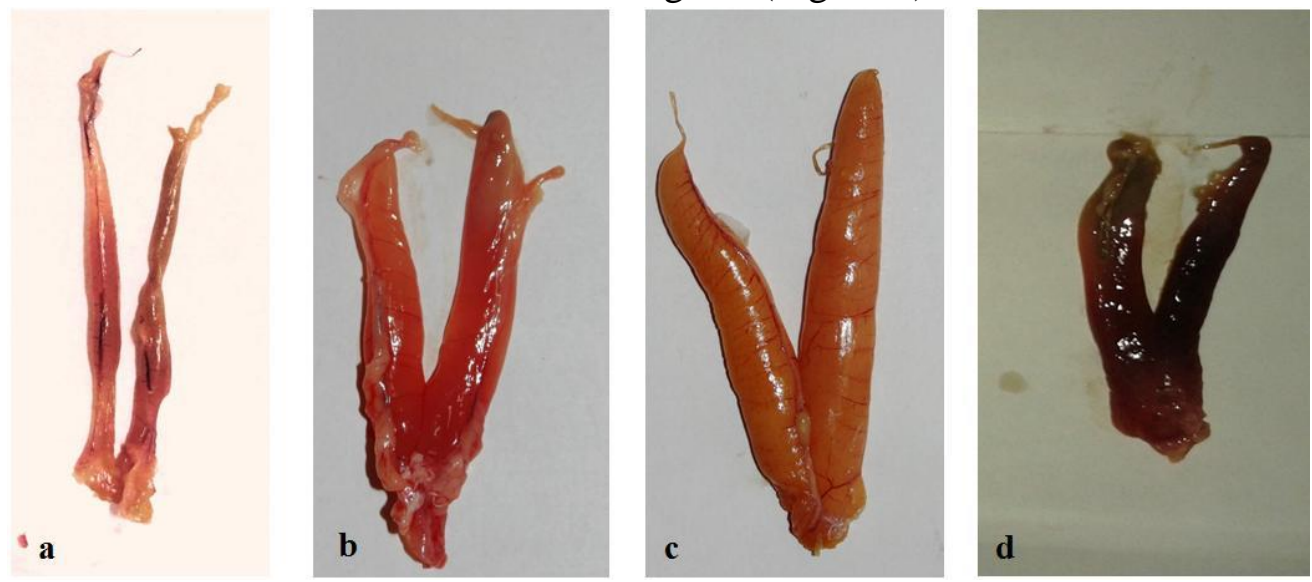

Figura 3. Estádios maturacionais de fêmeas de L. synagris: (a) imaturo; (b) em maturação; (c) maturo e (d) esvaziado. 
Tabela 3. Correlação entre os estágios macroscópicos e microscópios das fêmeas de L. synagris capturados nas proximidades da Raposa-MA no período de março de 2013 a fevereiro de 2014.

\begin{tabular}{|c|c|c|c|c|}
\hline \multirow{2}{*}{$\begin{array}{c}\text { Classificação } \\
\text { proposta }\end{array}$} & \multicolumn{3}{|c|}{ Aspectos macroscópicos } & \multirow{2}{*}{$\begin{array}{c}\begin{array}{c}\text { Aspectos } \\
\text { microscópicos }\end{array} \\
\begin{array}{c}\text { Ovócito } \\
\text { predominante }\end{array}\end{array}$} \\
\hline & $\begin{array}{c}\text { Aspectos dos } \\
\text { ovócitos } \\
\end{array}$ & Vascularização & $\begin{array}{c}\text { Coloração das } \\
\text { gônadas }\end{array}$ & \\
\hline \multirow{2}{*}{$\begin{array}{c}\text { Imaturo } \\
\text { Em maturação }\end{array}$} & - & Não & Translúcido & Fases I e II \\
\hline & $\begin{array}{c}\text { Esbranquiçados } \\
\text { pequenos e alguns } \\
\text { grandes }\end{array}$ & Pouca & Rosa claro & Fases II e III \\
\hline Maturo & $\begin{array}{c}\text { Amarelados } \\
\text { grandes }\end{array}$ & Elevada & Amarelo & Fase V \\
\hline Desovado & - & Hemorrágico & Vermelho & $\begin{array}{c}\text { Fases II, VI e } \\
\mathrm{AO}^{*}\end{array}$ \\
\hline
\end{tabular}

* Ocorre a presença dos ovócitos nas fases de cromatina; I: Fase de cromatina nucléolo; II: ovócitos perinucleolar; III: ovócitos em vitelogênese lipídica; IV: ovócitos em vitelogênese lipídica e protéica; V: vitelogênese completa; VI: em hialinização AO: ovócito atrésicos.
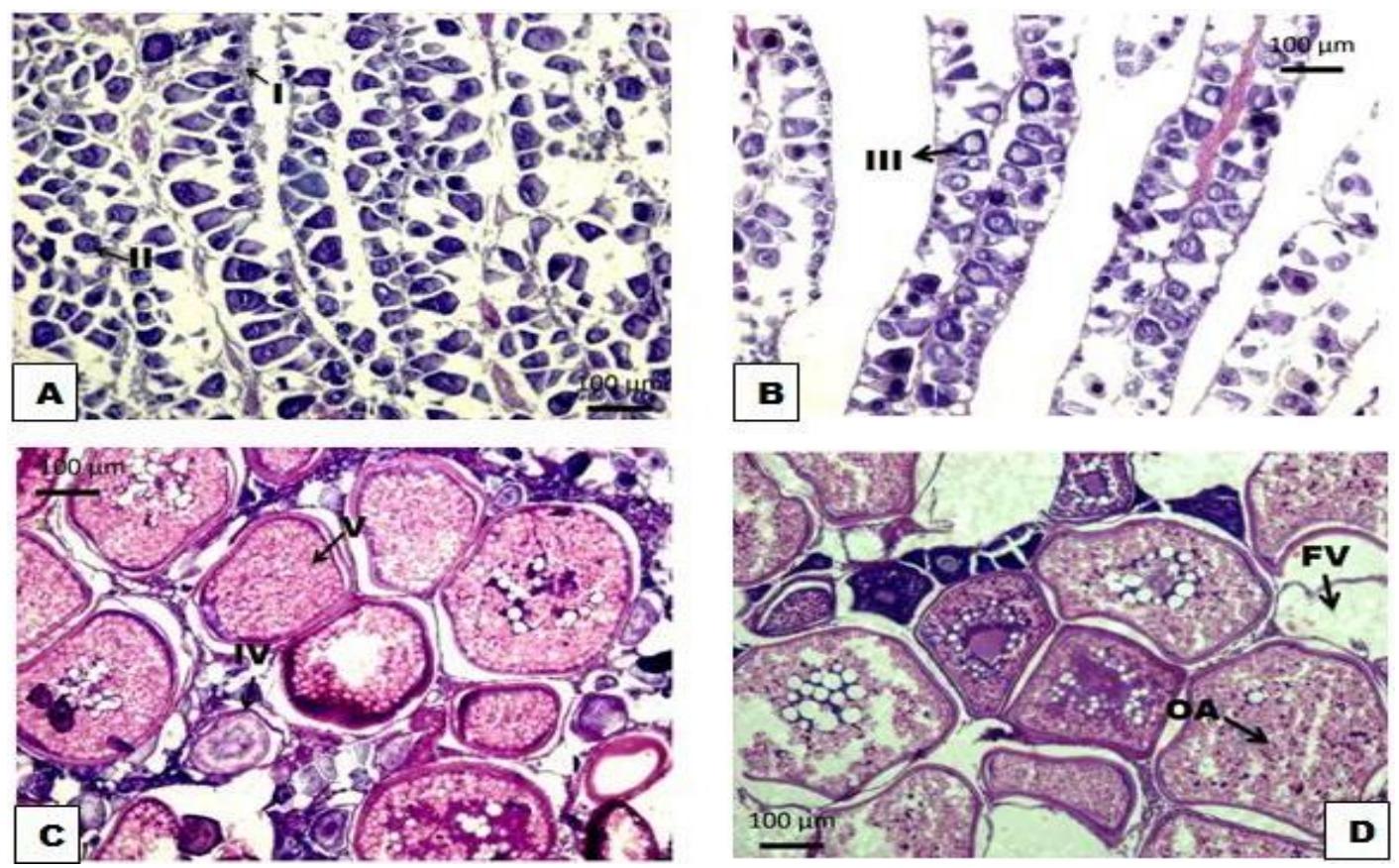

Figura 4. Fotomicrografia dos estádios de maturação gonadal das fêmeas de L.synagris. A: Ovário imaturo; B: Ovário em maturação; C: Ovário maturo; D: Ovário Desovado. HE 100X. Barra $=100 \mu \mathrm{m}$. I: Fase de cromatina nucléolo; II: ovócitos perinucleolar; III: ovócitos em vitelogênese lipídica; IV: ovócitos em vitelogênese lipídica e protéica; V: vitelogênese 
completa; FV: folículos vazios; OA: ovócito atrésicos.

Os valores médios de $\Delta \mathrm{RGS}$ e $\Delta \mathrm{K}$ para L. synagris foram significantemente diferentes entre os meses (Teste Kruskal-Wallis; $\mathrm{P}<0,05$ ) (Figura 5a e 5b), indicando que esta espécie apresenta desova parcelada com dois picos anuais: um período de atividade reprodutiva entre maio e junho e outro entre julho e agosto. Indivíduos maduros foram observados ao longo de quase todo o ano (Figura 5c), evidenciando que, apesar dos picos de maior atividade reprodutiva, L. synagris está apta a se reproduzir durante todo o ano. O tipo de desova parcelada foi confirmado pelos resultados obtidos na análise microscópica, uma vez que foi registrada a ocorrência nos ovários de ovócitos em várias fases do desenvolvimento ovocitário.
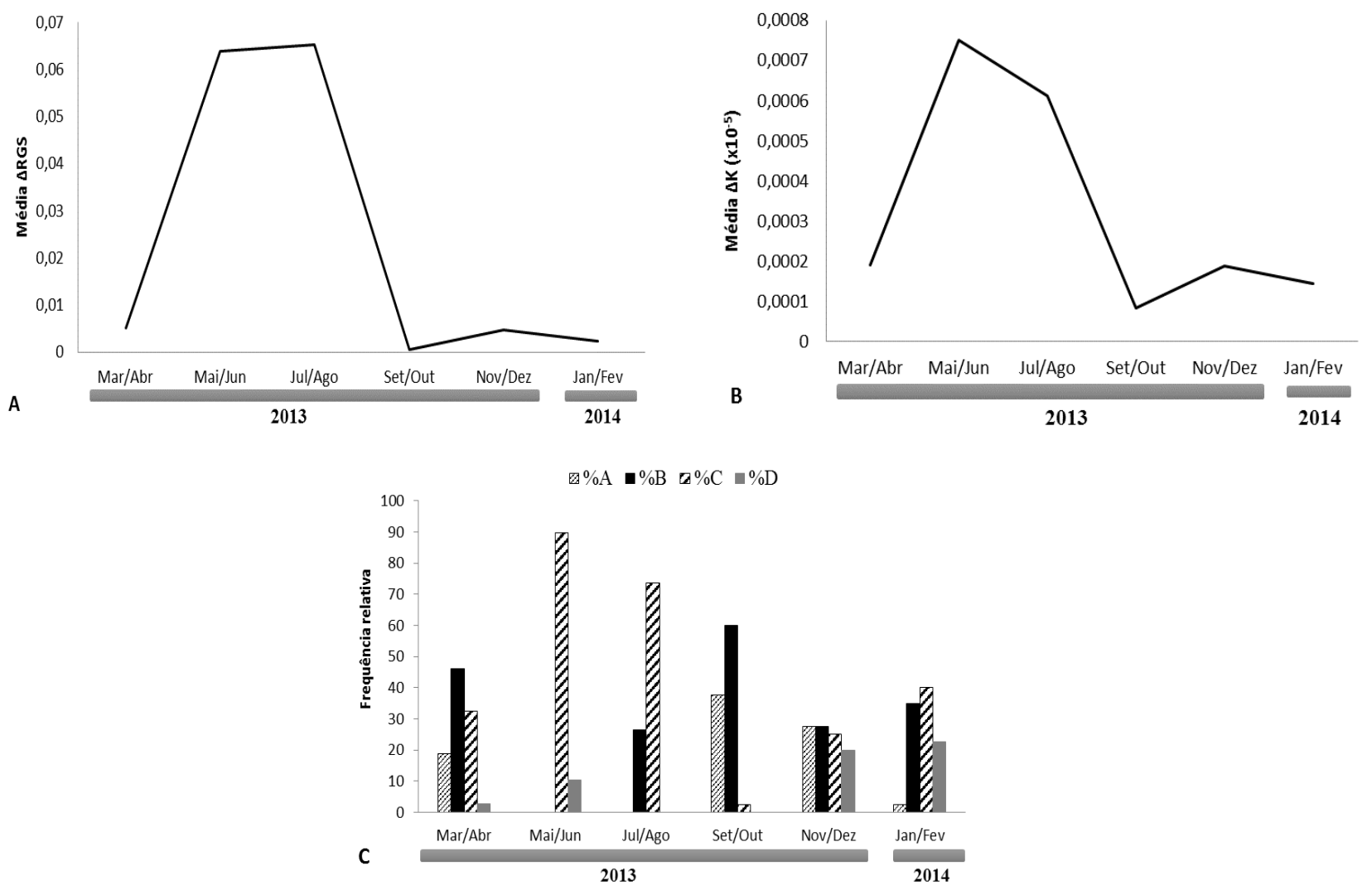

Figura 5. Valores médios do $\Delta$ RGS (A) e $\Delta \mathrm{K}$ (B) e frequência relativa bimestral dos estádios maturacionais de fêmeas (C) de L. synagris, capturados nas proximidades da Raposa-MA no período de março de 2013 a fevereiro de 2014.

A fecundidade absoluta média (FA), representada por aqueles ovócitos que, potencialmente, devem ser eliminados em uma próxima desova, teve uma variação de 457.931 a 954.976 ovócitos. Essa variação ocorreu em função do peso total e do comprimento total de cada individuo. A média da 
fecundidade absoluta foi de 709.271 ovócitos por desova.

\section{DisCUSSÃo}

Os exemplares de L. synagris no local estudado, apresentaram classes de comprimento total (fêmeas e machos 48,0 cm) superiores em relação a estudos com a mesma espécie em outros locais. Freitas, Moura, Francini-Filho \& Minte- Vera (2011) encontraram valor médio de 33,8 cm para fêmeas e 32,8 cm para machos no Banco de Abrolhos, essa diferença de tamanho pode dever-se aos tipos de artes de pesca utilizadas na captura dos espécimes, e com as características que cada local apresenta.

A relação peso/comprimento apresentou alometria do tipo negativa, resultados divergentes foram encontrados por Cavalcante, Oliveira \& Chellappa (2012) no litoral de Natal (Rio Grande do Norte, Brasil) para a mesma espécie, em que o ajuste encontrado foi do tipo alométrico positivo, tanto para machos como para fêmeas, o que pode ser explicado pelas distintas condições que cada local oferece no que se refere a disponibilidades de alimento e outros fatores ambientais relacionados ao habitat que podem estar influenciando no aumento da massa corpórea. Essas variáveis estão sob influência de diversos fatores, como: densidades populacionais, disponibilidade de alimento, $\mathrm{pH}$, temperatura e oxigênio, que por meio de interação entre eles, poderão afetar os valores estimados das variáveis da relação (SUASSUNA, 1999).

A proporção sexual encontrada para L. synagris foi de 4,74 fêmeas para cada 1 macho, havendo diferença significativa para o período amostrado. Para a mesma espécie no litoral de Natal (Rio Grande do Norte, Brasil) os machos predominaram na população com a proporção sexual (4,15M: 1F) diferindo significativamente $\left(\chi^{2}=23,88\right)$ (CAVALCANTE, OLIVEIRA \& CHELLAPPA, 2012) e no Banco de Abrolhos (Bahia, Brasil) a proporção sexual aproximou-se da esperada (1:1) (FREITAS, MOURA, FRANCINI-FILHO \& MINTE- VERA, 2011). O predomínio de fêmeas na população pode ser explicado por elas estarem mais adaptadas a eventos sucessivos, que atuam de forma distinta sobre os indivíduos de cada sexo (Vazzoler, 1996) e quanto à disponibilidade alimentar, que favorece o aumento na proporção de fêmeas quando o suprimento alimentar é adequado. Como no presente estudo foi detectada uma diferença significativa entre os sexos para o período amostrado, isso pode indicar uma possível segregação sexual para essa espécie na região analisada.

Gomez, Guzmán \& Chacón (2001), relataram que a maturidade sexual para L. synagris na Venezuela é atingida com aproximadamente $36,8 \mathrm{~cm}$ de comprimento. Para a mesma espécie no Banco de Abrolhos foi registrado L50 18,11 cm para fêmeas e 18,67 cm para machos (FREITAS, MOURA, FRANCINI-FILHO \& MINTE- VERA, 2011). No entanto, o presente resultado (L50 24,02 cm para as 
fêmeas e 23,59 cm nos machos) foi similar ao encontrado para a mesma espécie em Bermudas, onde o tamanho de maturação $50 \%$ foi estimado em 24,5 e 23,5 cm de comprimento zoológico para fêmeas e machos, respectivamente (LUCKHURST, DEAN \& REICHERT, 2000). Stearns \& Crandall (1984) e Wootton (1990) destacam que, dentro de uma mesma espécie, a idade e/ou o tamanho de maturação pode variar e que esta plasticidade seria determinada geneticamente e moldada pelas variáveis ambientais que se refletiria em diferentes taxas de crescimento e mortalidade, acarretando idade (ou tamanho) distinta de maturação.

A classificação macroscópica e microscópica dos diferentes estádios de maturação gonadal em peixes apresenta variações, de acordo com o autor considerado. Autores como Barbieri \& Santos (1988) classificam os estádios em cinco tipos, enquanto Teles \& Godinho (1997), em até seis. O presente trabalho seguiu a classificação de Vazzoler (1996) que divide os estádios em quatro tipos $(A, B, C, D)$. Tal variação de classificações é inerente às metodologias empregadas, refletindo em maior ou menor número de subdivisões.

A espécie L. synagris apresentou desova parcelada com dois picos anuais e foram observados indivíduos maduros ao longo de quase todo o ano. Ciclo reprodutivo ao longo de vários meses e picos de desova mais representativos no segundo semestre do ano foram observados por diversos autores para várias espécies de peixes, principalmente na América Central (RODRÍGUEZ, 1962; DRUZHININ, 1970; RIVERA-ARRIAGA, LARA- DOMINGUEZ, SANCHEZ-GIL \& YANEZARANCIBIA, 1996; GOMEZ, CHÁCON \& MÁRQUEZ 1999; LUCKHURST, DEAN \& REICHERT, 2000; GOMEZ, GUZMÁN \& CHACÓN, 2001). Este tipo de desova traz certa vantagem para a espécie em caso de impactos ambientais, pois garante novas oportunidades de reprodução. Esse fato mostra que a espécie aqui analisada está apta a se reproduzir durante o ano todo.

A determinação da fecundidade ou capacidade de produção de ovos em peixes é de grande relevância para o conhecimento do ciclo e história de vida de uma espécie (GOMEZ, GUZMÁN \& CHACÓN, 2001). A fecundidade de peixes é diretamente relacionada com fatores ambientais e condições fisiológicas individuais. O desenvolvimento gonadal indica que a espécie completa todo seu ciclo de vida na área em estudo. Outro fator que confirma essa indicação é a presença marcante de peixes imaturos dessa espécie na área analisada, sendo que a entrada destes novos recrutas é consequência do processo reprodutivo nos meses anteriores. 


\section{CONCLUSÕES}

L. synagris está apta a reproduzir o ano todo, no entanto, para elaboração de medidas de manejo para a espécie é importante restringir a pesca nestas localidades, durante o período de maio a agosto, bem como estipular o tamanho mínimo de captura das fêmeas de $24,02 \mathrm{~cm}$ de comprimento como forma de proteger o estoque desovante da população proporcionando a conservação e o uso sustentável deste recurso e podendo assegurar seus estoques naturais para futuras gerações. Com isso, essa região é uma área de reprodução e crescimento para a espécie analisada.

\section{REFERÊNCIAS}

BARBIERI, G. \& SANTOS, E.P. DOS S. (1988). Análise comparativa do crescimento e de aspectos reprodutivos da piava, Leporinus friderici (Bloch, 1794) (Osteichthyes, Anostomidae) da represa do Lobo e do rio Mogi-Guaçu, Estado de São Paulo. Ciência e Cultura, 40(7): 693-697.

BEGOSSI, A.; SALIVONCHYK, S.V.; ARAUJO, L.G., ANDREOLI, T.B., CLAUZET, M., MARTINELli, C.M., FERREIRA, A.G.L., OLIVEIRA, L.E.C. \& SILVANO, R.A.M. (2011). Ethnobiology of snappers (Lutjanidae): target species and suggestions for management. Journal of Ethnobiology and Ethnomedicine, 7: 11.

BRASIL, MINISTÉRIO DA PESCA E AQUICULTURA (MPA). (2010). Boletim Estatístico da Pesca e Aquicultura: Brasil 2008-2009. Brasília.

BRASIL, MINISTÉRIO DA PESCA E AQUICULTURA (MPA). (2011) Boletim Estatístico da Pesca e Aquicultura 2011. Brasília.

CAVALCANTE, L.D.F.D.M.; OLIVEIRA, M. R. \& CHELLAPPA, S. (2012). Aspectos reprodutivos do ariacó, Lutjanus synagris nas águas costeiras do Rio Grande do Norte. Biota Amazônia, 2(1): 45-50.

DRUZHININ, A. (1970). The range and biology of snapper (Familia Lutjanidae). Journal Ichthyology, 10: $175-735$.

FIGUEIREDO, J.L. \& MENEZES, N.A. (2000). Manual de peixes marinhos do Sudeste do Brasil. São Paulo: Museu de Zoologia/USP.

FRÉDOU, T.; FERREIRA, B.P. \& LETOURNNEUR, Y. (2006). A univariate and multivariate study of reef fishes of northeastern Brazil. ICES Journal of Marine Sciences, 63: 883-896.

FREITAS, M.O.; MOURA, R.L.; FRANCINI-FILHO, R.B. \& MINTE-VERA, C.V. (2011). Spawning patterns of commercially important reef fish (Lutjanidae and Serranidae) in the tropical western South Atlantic. Scientia Marina, 75(1): 135-146.

GÓMEZ, G.; GUZMÁN, R.; CHACÓN, R. \& MÁRQUEZ, M. (1999). Talla de madurez y fecundidad del pargo guanapo, Lutjanus synagris en el Golfo de Paria Edo. Sucre, Venezuela. Durante el período 1998. Acta Científica Venezoelana, 50(2): 375. 
GÓMEZ, G.; GUZMÁN, R. \& CHACÓN, R. (2001) Parámetros reproductivos y poblacionales de Lutjanus synagris em el Golfo de Paria, Venezuela. Zootecnia Tropical, 19(3): 335-357.

IBAMA. ESTATÍSTICA DA PESCA 2007. (2008). Brasil: Grandes Regiões e Unidades da Federação. Brasília.

IBAMA. (2010). Gestão do uso dos recursos pesqueiros marinhos no Brasil/ José Dias Neto. $2^{\mathrm{a}}$ ed. Brasília.

IBGE - Instituto Brasileiro de Geografia e Estatística (2014). Acessado em 10 de dezembro de 2014 emhttp://www.cidades.ibge.gov.br/xtras/perfil.php?lang=\&codmun=210945\&search=maranhao|raposa| infograficos:-informacoes-completas.

KING, M. (1995). Fisheries biology: assessment and management. Oxford, Fishing News Books.

LUCKHURST, B. E.; DEAN, J. M. \& REICHERT, M. (2000). Age, growth and reproduction of the lane snapper Lutjanus synagris (Pisces: Lutjanidae) at Bermuda. Marine Ecology Progress Series, 203: 255-261.

MCEACHRAN, J.D. \& FECHHELM, J.D. (2005). Fishes of the Gulf of Mexico. Austin: University of Texas Press.

MENEZES, N.A.; BUCKUP, P.A.; FIGUEIREDO, J.L. \& MOURA, R.L. (2003). Catálogo das espécies de peixes marinhos do Brasil. São Paulo: Museu de Zoologia Universidade de São Paulo.

NELSON, J.S. (1994). Fishes of the world. $3^{\circ}$ ed. Inc. New York: John Wiley \& Sons.

ORTIZ-ORDÓNEZ, E.; URÍA GALICIA1, E.; LÓPEZ-LÓPEZ, E.; MAYA, J.P. \& CARVAJAL HERNÁNDEZ, A.L. (2007). Reproductive cycle by histological characterization of the ovary in the butterfly goodeid Ameca splendens from the upper Rio Ameca Basin, México. Journal of Applied Ichthyology, 23: 40-45.

RIVERA-ARRIAGA, E.; LARA-DOMINGUEZ, A.L.; RAMOS-MIRANDA, J.; SANCHEZ-GIL, P, \& YANEZ-ARANCIBIA, A. (1996). Ecology and population dynamics of Lutjanus synagris on Campeche Bank. In: Biology, fisheries and culture of tropical groupers and snapper, 48: 11-18.

RODRÍGUEZ, P. (1962). Estudios estadísticos y biológicos sobre la biajaiba (Lutjanus synagris) de La plataforma sur oriental de Cuba. Ibiden. 4: 1-99.

SOUZA, L. DE L. G.; CHELLAPPA, S. \& GURGEL, H. DE C. B. (2007). Biologia reprodutiva do peixe-donzela, Stegastes fuscus Cuvier, em arrecifes rochosos no nordeste do Brasil. Revista Brasileira de Zoologia, 24(2): 419-425.

STEARNS, S.C. \& CRANDALL. (1984). Plasticity for age at sexual maturity: a life-hitory responses to inavoidable stress. In: Potts, G. W. \& Wootton, R.j ads. Fish reproduction: Strategies and tactics. Academic Press. 13-33. 
SUASSUNA, J. (1999). Contribuição ao estudo hidrológico de semi-árido nordestino. Recife, Fundação Joaquim Nabuco.

TELES, M.E.O. \& GODINHO,H.P. (1997). Ciclo reprodutivo da pirambeba Sertrasalmus brandtii (Teleostei: Characidae) na represa de Três Marias, Rio São Francisco. Revista Brasileira de Biologia, 57: $177-184$.

VAZZOLER, A.E.A.M. (1981). Manual de métodos para estudos biológicos para populações de peixes, reprodução e crescimento. Brasília, CNPq. Programa Nacional de Zoologia.

VAZZOLER, A.E.A DE M. (1996). Biologia da Reprodução dos Teleósteos: teoria e prática. São Paulo: SBI/EDUEM.

WOOTTON, R. J. (1990). Ecology of teleost fishies. London, Chapman and Hall.

ZAR, J. H. (1996). Biostatistical analysis. $3^{\circ}$ ed. Ed. Prentice-Hall International INC. 